\title{
Two-level preconditioners for the Helmholtz equation
}

\author{
Marcella Bonazzoli ${ }^{1}$, Victorita Dolean ${ }^{1,2}$, Ivan G. Graham ${ }^{3}$, Euan A. \\ Spence $^{3}$, and Pierre-Henri Tournier ${ }^{4}$
}

\section{Introduction}

Solving the Helmholtz equation $-\Delta u-k^{2} u=f$ is a challenging task because of its indefinite nature and its highly oscillatory solution when the wavenumber $k$ is high. Although there have been different attempts to solve it efficiently, we believe that there is no established and robust preconditioner, whose behavior is independent of $k$, for general decompositions into subdomains. In Conen et al. [2014] a two-level preconditioner was introduced, where the coarse correction involves local eigenproblems of Dirichletto-Neumann (DtN) maps. This method proved to be very robust with respect to heterogeneous coefficients compared to the reference preconditioner based on plane waves, and its construction is completely automatic without the need for parameter tuning. Another method was developed in Graham et al. [2017b,a], where two-level domain decomposition approximations of the Helmholtz equation with absorption $-\Delta u-\left(k^{2}+i \varepsilon\right) u=f$ were used as preconditioners for the pure Helmholtz equation without absorption; there the coarse correction is based on a coarse mesh with diameter constrained by $k$. Our purpose is to compare numerically the performance of the latter with the two-level method based on DtN maps, both in two and three dimensions.

\section{Definition of the problem}

Consider the interior Helmholtz problem of the following form: let $\Omega \subset \mathbb{R}^{d}$, $d=2,3$, be a polyhedral, bounded domain; find $u: \Omega \rightarrow \mathbb{C}$ such that

\footnotetext{
1 Université Côte d'Azur, CNRS, LJAD, France, e-mail: marcella.bonazzoli@unice.fr

${ }^{2}$ University of Strathclyde, Glasgow, UK, e-mail: Victorita.Dolean@strath.ac.uk

3 University of Bath, UK, e-mail: I.G.Graham@bath.ac.uk, E.A.Spence@bath.ac.uk

${ }^{4}$ UPMC Univ Paris 06, LJLL, Paris, France, e-mail: tournier@ljll.upmc.fr
} 


$$
\begin{aligned}
-\Delta u-\left(k^{2}+\mathbf{i} \varepsilon\right) u & =f & & \text { in } \Omega, \\
\frac{\partial u}{\partial n}-\mathbf{i} \eta u & =0 & & \text { on } \Gamma=\partial \Omega .
\end{aligned}
$$

The wavenumber $k$ is given by $k(\mathbf{x})=\omega / c(\mathbf{x})$, where $\omega$ is the angular frequency and $c$ is the speed of propagation that might depend on $\mathbf{x} \in \Omega$; we take $\eta=\operatorname{sign}(\varepsilon) k$ if $\varepsilon \neq 0, \eta=k$ if $\varepsilon=0$, as Robin boundary condition parameter. We are interested in solving the problem in the case $\varepsilon=0$, using $\varepsilon$ as a parameter when building the preconditioner. The variational formulation of Problem (1) is: find $u \in V=H^{1}(\Omega)$ such that $a_{\varepsilon}(u, v)=F(v), \forall v \in V$, where $a_{\varepsilon}(.,):. V \times V \rightarrow \mathbb{C}$ and $F: V \rightarrow \mathbb{C}$ are defined by

$$
a_{\varepsilon}(u, v)=\int_{\Omega}\left(\nabla u \cdot \overline{\nabla v}-\left(k^{2}+\mathrm{i} \varepsilon\right) u \bar{v}\right)-\int_{\Gamma} \mathrm{i} \eta u \bar{v}, \quad F(v)=\int_{\Omega} f \bar{v} .
$$

Note that if $\varepsilon \neq 0$ and $\eta=\operatorname{sign}(\varepsilon) k, a_{\varepsilon}$ is coercive (see $\S 2$ in Graham et al. [2017b]). We consider a discretization of the variational problem using piecewise linear finite elements on a uniform simplicial mesh $\mathcal{T}_{h}$ of $\Omega$. Denoting by $V_{h} \subset V$ the corresponding finite element space and by $\left\{\phi_{k}\right\}_{k=1}^{n}$ its basis functions, $n:=\operatorname{dim}\left(V_{h}\right)$, the discretized problem reads: find $u_{h} \in V_{h}$ such that $a_{\varepsilon}\left(u_{h}, v_{h}\right)=F\left(v_{h}\right), \forall v_{h} \in V_{h}$, that is, in matrix form,

$$
A_{\varepsilon} \mathbf{u}=\mathbf{f},
$$

where the coefficients of the matrix $A_{\varepsilon} \in \mathbb{C}^{n \times n}$ and the right-hand side $\mathbf{f} \in \mathbb{C}^{n}$ are given by $\left(A_{\varepsilon}\right)_{k, l}=a\left(\phi_{l}, \phi_{k}\right)$ and $(\mathbf{f})_{k}=F\left(\phi_{k}\right)$. The matrix $A_{\varepsilon}$ is complex, symmetric (but not Hermitian), and indefinite if $\varepsilon=0$.

\section{Two-level domain decomposition preconditioners}

In the following we will define the domain decomposition preconditioners for the linear system $A_{0} \mathbf{u}=\mathbf{f}$ resulting from the discretization of the Helmholtz problem without absorption $(\varepsilon=0)$. These are two-level Optimized Restricted Additive Schwarz (ORAS) algorithms, where "optimized" refers to the use of Robin boundary conditions at the interface between subdomains. In the terminology of Graham et al. [2017b], the prefix O is replaced with Imp, which stands for impedance (i.e. Robin) boundary conditions.

First of all, consider a decomposition of the domain $\Omega$ into a set of overlapping subdomains $\left\{\Omega_{j}\right\}_{j=1}^{N_{\text {sub }}}$, with each subdomain consisting of a union of elements of the mesh $\mathcal{T}_{h}$. Let $V_{h}\left(\Omega_{j}\right)=\left\{\left.v\right|_{\Omega_{j}}: v \in V_{h}\right\}, 1 \leq j \leq N_{\text {sub }}$, denote the space of functions in $V_{h}$ restricted to the subdomain $\Omega_{j}$. Let $n_{j}$ be the dimension of $V_{h}\left(\Omega_{j}\right), 1 \leq j \leq N_{\text {sub. For }} 1 \leq j \leq N_{\text {sub }}$, we define a restriction operator $\mathcal{R}_{j}: V_{h} \rightarrow V_{h}\left(\Omega_{j}\right)$ by injection, i.e. for $u \in V_{h}$ we set $\left(\mathcal{R}_{j} u\right)\left(\mathbf{x}_{i}\right)=u\left(\mathbf{x}_{i}\right)$ for all $\mathbf{x}_{i} \in \Omega_{j}$. We denote by $R_{j}$ the corresponding 
Boolean matrix in $\mathbb{R}^{n_{j} \times n}$ that maps coefficient vectors of functions in $V_{h}$ to coefficient vectors of functions in $V_{h}\left(\Omega_{j}\right)$. Let $D_{j} \in \mathbb{R}^{n_{j} \times n_{j}}$ be a diagonal matrix corresponding to a partition of unity in the sense that $\sum_{i=1}^{N_{\text {sub }}} \tilde{R}_{i}^{T} R_{i}=I$, where $\tilde{R}_{j}:=D_{j} R_{j}$. Then the one-level ORAS preconditioner (which is also the one-level ImpRAS of Graham et al. [2017b]) reads

$$
M_{1, \varepsilon}^{-1}:=\sum_{j=1}^{N_{\text {sub }}} \tilde{R}_{j}^{T} A_{j, \varepsilon}^{-1} R_{j} .
$$

We define the matrices $A_{j, \varepsilon}$ in (3) to be the matrices stemming from the discretization of the following local Robin problems with absorption

$$
\begin{aligned}
-\Delta u_{j}-\left(k^{2}+i \varepsilon\right) u_{j} & =f & & \text { in } \Omega_{j}, \\
\frac{\partial u_{j}}{\partial n_{j}}-i \eta u_{j} & =0 & & \text { on } \partial \Omega_{j} .
\end{aligned}
$$

In order to achieve weak dependence on the wavenumber $k$ and number of subdomains, we add a coarse component to (3). The two-level preconditioner can be written in a generic way as follows

$$
M_{2, \varepsilon}^{-1}=Q M_{1, \varepsilon}^{-1} P+Z E^{-1} Z^{*},
$$

where $*$ denotes the conjugate transpose, $M_{1, \varepsilon}^{-1}$ is the one-level preconditioner (3), $Z$ is a rectangular matrix with full column rank, $E=Z^{*} A_{\varepsilon} Z$ is the so-called coarse grid matrix, $\Xi=Z E^{-1} Z^{*}$ is the so-called coarse grid correction matrix. If $P=Q=I$ this is an additive two-level preconditioner (which would be called two-level ImpRAS in Graham et al. [2017b]). If $P=I-A_{\varepsilon} \Xi$ and $Q=I-\Xi A_{\varepsilon}$, this is a hybrid two-level preconditioner (ImpHRAS in Graham et al. [2017b]), also called the Balancing Neumann Neumann (BNN) preconditioner. Preconditioner (4) is characterized by the choice of $Z$, whose columns span the coarse space (CS). We will consider the following two cases:

The grid coarse space The most natural coarse space would be one based on a coarser mesh, we subsequently call it "grid coarse space". Let us consider $\mathcal{T}_{H_{\text {coarse }}}$ a simplicial mesh of $\Omega$ with mesh diameter $H_{\text {coarse }}$ and $V_{H_{\text {coarse }}} \subset V$ the corresponding finite element space. Let $\mathcal{I}_{0}: V_{H_{\text {coarse }}} \rightarrow V_{h}$ be the nodal interpolation operator and define $Z$ as the corresponding matrix. Then in this case $E=Z^{*} A_{\varepsilon} Z$ is really the stiffness matrix of the problem (with absorption) discretized on the coarse mesh. Related preconditioners without absorption are used in Kimn and Sarkis [2007].

The DtN coarse space This coarse space (see Conen et al. [2014]) is based on local Dirichlet-to-Neumann (DtN) eigenproblems on the subdomain interfaces. For a subdomain $\Omega_{i}$, first of all consider $a^{(i)}: H^{1}\left(\Omega_{i}\right) \times H^{1}\left(\Omega_{i}\right) \rightarrow \mathbb{R}$ 


$$
a^{(i)}(v, w)=\int_{\Omega_{i}}\left(\nabla v \cdot \overline{\nabla w}-\left(k^{2}+\mathrm{i} \varepsilon\right) v \bar{w}\right)-\int_{\partial \Omega_{i} \cap \partial \Omega} \mathbf{i} \eta u \bar{v} .
$$

Let $\left(A^{(i)}\right)_{k l}=a^{(i)}\left(\phi_{k}, \phi_{l}\right)$, and let $I$ and $\Gamma_{i}$ be the sets of indices corresponding, resp., to the interior and boundary dofs on $\Omega_{i}$, with $n_{I}$ and $n_{\Gamma_{i}}$ their cardinalities. With the usual block notation, the subscripts $I$ and $\Gamma_{i}$ for the matrices $A$ and $A^{(i)}$ denote the entries of these matrices associated with the respective dofs. Let $M_{\Gamma_{i}}=\left(\int_{\Gamma_{i}} \phi_{k} \phi_{l}\right)_{k, l \in \Gamma_{i}}$ be the mass matrix on the interface $\Gamma_{i}=\partial \Omega_{i} \backslash \partial \Omega$ of subdomain $\Omega_{i}$. We need to solve the following eigenproblem: find $(\mathbf{u}, \lambda) \in \mathbb{C}^{n_{\Gamma_{i}}} \times \mathbb{C}$, s.t.

$$
\left(A_{\Gamma_{i} \Gamma_{i}}^{(i)}-A_{\Gamma_{i} I} A_{I I}^{-1} A_{I \Gamma_{i}}\right) \mathbf{u}=\lambda M_{\Gamma_{i}} \mathbf{u} .
$$

Now, the matrix $Z$ of the DtN coarse space is a rectangular, block-diagonal matrix with blocks $W_{i}$, associated with the subdomain $\Omega_{i}, 1 \leq i \leq N_{\text {sub }}$, given by Algorithm 3.1. If $m_{i}$ is the number of eigenvectors selected by the automatic criterion in Line 2 of Algorithm 3.1, the block $W_{i}$ has dimensions $n_{i} \times m_{i}$, and the matrix $Z$ has dimensions $n \times \sum_{j=1}^{N_{\text {sub }}} m_{i}$. Due to the overlap in the decomposition, the blocks may share some rows inside the matrix $Z$.

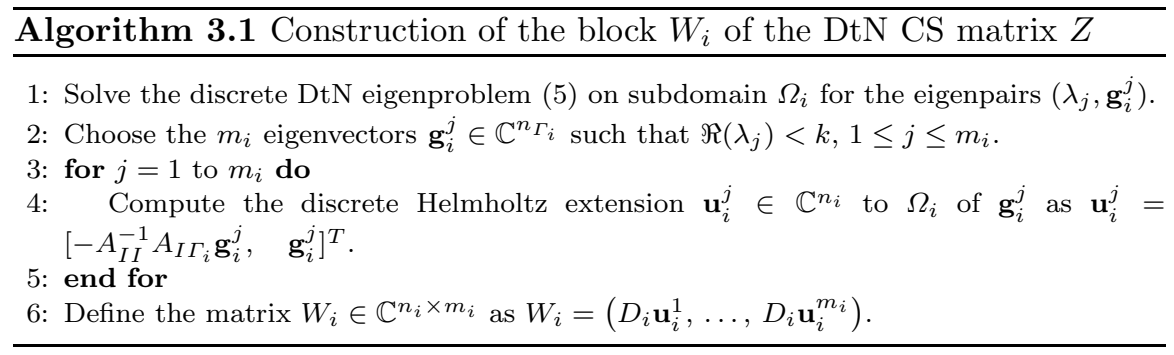

\section{Numerical experiments}

We solve (2) with $\varepsilon=0$ on the unit square/cube, with a uniform simplicial mesh of diameter $h \sim k^{-3 / 2}$, which is believed to remove the pollution effect. The right-hand side is given by $f=-\exp \left(-100\left((x-0.5)^{2}+(y-0.5)^{2}\right)\right)$ for $d=2, f=-\exp \left(-400\left((x-0.5)^{2}+(y-0.5)^{2}+(z-0.5)^{2}\right)\right)$ for $d=3$.

We use GMRES with right preconditioning (with a tolerance $\tau=10^{-6}$ ), starting with a random initial guess, which ensures, unlike a zero initial guess, that all frequencies are present in the error; the stopping criterion is based on the relative residual. We consider a regular decomposition into subdomains (squares/cubes), the overlap for each subdomain is of size $\mathcal{O}(2 h)$ in all directions and the two-level preconditioner (4) is used in the hybrid 
way. All the computations are done in the open source language FreeFem ++ (http://www.freefem.org/fft+/). The $3 d$ code is parallelized and run on the TGCC Curie supercomputer. We assign each subdomain to one processor. So in our experiments the number of processors increases if the number of subdomains increases. To apply the preconditioner, the local problems in each subdomain (with matrices $A_{j, \varepsilon}$ in (3)) and the coarse space problem (with matrix $E$ in (4)) are solved with a direct solver.

As in Graham et al. [2017b,a], in the experiments we take the subdomain diameter $H_{\text {sub }}$ and the coarse mesh diameter $H_{\text {coarse }}$ constrained by $k$ : $H_{\text {sub }} \sim k^{-\alpha}$ and $H_{\text {coarse }} \sim k^{-\alpha^{\prime}}$, for some choices of $0<\alpha, \alpha^{\prime}<=1$ detailed in the following; if not differently specified, we take $\alpha=\alpha^{\prime}$, which is the setting of all numerical experiments in Graham et al. [2017b]. Note that $H_{\text {coarse }}$ does not appear as a parameter in the DtN coarse space. We denote by $n_{\mathrm{CS}}$ the size of the coarse space. For the grid coarse space $n_{\mathrm{CS}}=\left(1 / H_{\text {coarse }}+1\right)^{d}$, the number of dofs for the nodal linear finite elements in the unit square/cube. For the DtN coarse space $n_{\mathrm{CS}}=\sum_{j=1}^{N_{\text {sub }}} m_{i}$, the total number of computed eigenvectors for all the subdomains. While we solve the pure Helmholtz problem without absorption, both the one-level preconditioner (3) and the twolevel preconditioner (4) are built from problems which can have non zero absorption given by $\varepsilon_{\text {prec }}=k^{\beta}$. In the experiments we put $\beta=1$ or $\beta=2$.

In the following tables we compare the one-level preconditioner, the twolevel preconditioners with the grid coarse space and with the DtN coarse space in terms of number of iterations of GMRES and size of the coarse space $\left(n_{\mathrm{CS}}\right)$, for different values of the wavenumber $k$ and of the parameters $\alpha, \beta$. We also report the number of subdomains $N_{\text {sub }}$, which is controlled by $k$ and $\alpha$ as mentioned above. Since $h \sim k^{-3 / 2}$, the dimension $n$ of the linear system matrix is of order $k^{3 d / 2}$; for 3 d experiments we report $n$ explicitly. Tables 1, 2 concern the $2 d$ problem, Table 3 the $3 d$ problem.

In Table 1, we let the DtN coarse space size be determined by the automatic choice criterion in Line 2 of Algorithm 3.1 (studied in Conen et al. [2014]) and the grid coarse space size by $H_{\text {coarse }} \sim k^{-\alpha}$. We see that the $\mathrm{DtN}$ coarse space is much larger than the grid coarse space and gives fewer iterations. The preconditioners with absorption $\varepsilon_{\text {prec }}=k^{2}$ perform much worse than those with absorption $\varepsilon_{\text {prec }}=k$ independently of $n_{\mathrm{CS}}$. For $\varepsilon_{\text {prec }}=k$, when $\alpha=1$ the number of iterations grows as $k^{0.9}$, respectively $k^{1.1}$, for the grid coarse space, respectively DtN coarse space (excluding the first two values for $k$ small where the asymptotic behaviour is not reached yet) while the one-level preconditioner performs poorly (for $k=80$ it needs more than 500 iterations to converge). When $\alpha<1$, i.e. for coarser coarse meshes, the growth with $k$ is higher, and for $\alpha=0.6$ the two-level preconditioner is not much better than the one-level preconditioner because the coarse grid problem is too coarse; for $\alpha=0.8$ with the DtN coarse space the growth with $k$ degrades less than with the grid coarse space.

We have seen in Table 1 that the DtN coarse space gives fewer iterations than the grid coarse space, but their sizes differed significantly. Therefore, in 


\begin{tabular}{|c|c|c|c|c|c|c|c|c|c|c|c|}
\hline & \multicolumn{5}{|c|}{$\beta=1$} & \multicolumn{5}{|c|}{$\beta=2$} \\
\hline & & \multicolumn{5}{|c|}{$\alpha=0.6$} & \multicolumn{5}{|c|}{$\alpha=0.6$} \\
\hline$k$ & $N_{\text {sub }}$ & 1-level & grid CS & $n_{\mathrm{CS}}$ & DtN CS & $n_{\mathrm{CS}}$ & 1-level & grid CS & $n_{\mathrm{CS}}$ & DtN CS & $n_{\mathrm{CS}}$ \\
\hline 10 & 9 & 22 & 19 & 16 & 11 & 39 & 28 & 27 & 16 & 23 & 40 \\
\hline 20 & 36 & 48 & 46 & 49 & 26 & 204 & 67 & 56 & 49 & 40 & 220 \\
\hline 40 & 81 & 78 & 98 & 100 & 37 & 531 & 121 & 114 & 100 & 72 & 578 \\
\hline 60 & 121 & 109 & 114 & 144 & 43 & 1037 & 169 & 165 & 144 & 109 & 920 \\
\hline \multirow{2}{*}{80} & 169 & 139 & 138 & 196 & 93 & 1588 & 223 & 216 & 196 & 126 & 1824 \\
\hline & & \multicolumn{5}{|c|}{$\alpha=0.8$} & \multicolumn{5}{|c|}{$\alpha=0.8$} \\
\hline$k$ & $N_{\text {sub }}$ & 1-level & grid CS & $n_{\mathrm{CS}}$ & DtN CS & $n_{\mathrm{CS}}$ & 1-level & grid CS & $n_{\mathrm{CS}}$ & DtN CS & $n_{\mathrm{CS}}$ \\
\hline 10 & 36 & 35 & 19 & 49 & 10 & 122 & \begin{tabular}{|l|}
39 \\
\end{tabular} & 27 & 49 & 28 & 86 \\
\hline 20 & 100 & 71 & 35 & 121 & 13 & 394 & 83 & 51 & 121 & 41 & 362 \\
\hline 40 & 361 & 158 & 88 & 400 & 22 & 1440 & 182 & 95 & 400 & 71 & 1370 \\
\hline 60 & 676 & 230 & 187 & 729 & 39 & 2700 & 268 & 150 & 729 & 103 & 2698 \\
\hline \multirow[t]{2}{*}{80 . } & 1089 & 304 & 331 & 1156 & 68 & 4352 & 355 & 214 & 1156 & 138 & 4350 \\
\hline & & \multicolumn{5}{|c|}{$\alpha=1$} & \multicolumn{5}{|c|}{$\alpha=1$} \\
\hline$k$ & $N_{\text {sub }}$ & 1-level & grid CS & $n_{\mathrm{CS}}$ & DtN CS & $n_{\mathrm{CS}}$ & 1-level & grid CS & $n_{\mathrm{CS}}$ & DtN CS & $n_{\mathrm{CS}}$ \\
\hline 10 & \begin{tabular}{|l|l}
100 \\
\end{tabular} & 65 & 26 & 121 & 11 & 324 & 57 & 30 & 121 & 23 & 324 \\
\hline 20 & 400 & 122 & 26 & 441 & 14 & 1120 & 130 & 49 & 441 & 42 & 1120 \\
\hline 40 & 1600 & 286 & 33 & 1681 & 20 & 4640 & 296 & 80 & 1681 & 72 & 4640 \\
\hline 60 & 3600 & 445 & 45 & 3721 & 29 & 10560 & 455 & 112 & 3721 & 101 & 10560 \\
\hline 80 & 6400 & $>500$ & 62 & 6561 & 44 & 18880 & $>500$ & 149 & 6561 & 134 & 18880 \\
\hline
\end{tabular}

Table 1: $(d=2)$ Number of iterations (and coarse space size $\left.n_{\mathrm{CS}}\right)$ for the onelevel preconditioner and the two-level preconditioners with the grid coarse space/DtN coarse space, with $H_{\text {sub }}=H_{\text {coarse }} \sim k^{-\alpha}, \varepsilon_{\text {prec }}=k^{\beta}$.

Table 2 we compare the two methods forcing $n_{\mathrm{CS}}$ to be similar. On the left, we force the DtN coarse space to have a smaller size, similar to the one of the grid coarse space, by taking just $m_{i}=2$ eigenvectors for each subdomain. On the right, we do the opposite, we force the grid coarse space to have the size of the DtN coarse space obtained in Table 1, by prescribing a smaller coarse mesh diameter $H_{\text {coarse, }}$ while keeping the same number of subdomains as in Table 1 with $H_{\text {sub }} \sim k^{-\alpha}$. We can observe that for smaller coarse space sizes (left) the grid coarse space gives fewer iterations than the DtN coarse space, while for larger coarse space sizes (right) the result is reversed.

We have seen that the coarse mesh obtained with $H_{\text {coarse }} \sim k^{-\alpha^{\prime}}, \alpha^{\prime}=\alpha$ can be too coarse if $\alpha=0.6$. At the same time, for $\alpha=1$ the number of subdomains gets quite large since $H_{\text {sub }} \sim k^{-\alpha}$, especially in $3 d$; this is not desirable because in our parallel implementation we assign each subdomain to one processor, so communication among them would prevail and each processor would not be fully exploited since the subdomains would become very small. Therefore, to improve convergence with the grid coarse space while maintaining a reasonable number of subdomains, we consider separate $H_{\text {coarse }}$ and $H_{\text {sub }}$, taking $\alpha^{\prime} \neq \alpha$. For load balancing (meant as local problems having the same size as the grid coarse space problem), in $3 d$ we choose $\alpha^{\prime}=3 / 2-\alpha$. The DtN coarse space is still built by keeping the eigenvectors verifying the 


\begin{tabular}{|c|c|c|c|c|c|c|c|c|c|}
\hline & \multicolumn{4}{|c|}{$n_{\mathrm{CS}}$ forced by grid CS } & \multicolumn{4}{|c|}{$n_{\mathrm{CS}}$ forced by DtN CS } \\
\hline & & \multicolumn{4}{|c|}{$\alpha=0.6$} & \multicolumn{4}{|c|}{$\alpha=0.6$} \\
\hline$k$ & $N_{\text {sub }}$ & grid CS & $n_{\mathrm{CS}}$ & DtN CS & $n_{\mathrm{CS}}$ & grid CS & $n_{\mathrm{CS}}$ & DtN CS & $n_{\mathrm{CS}}$ \\
\hline 10 & 9 & 19 & 16 & 18 & 18 & 17 & 36 & 11 & 39 \\
\hline 20 & 36 & 46 & 49 & 44 & 72 & 24 & 196 & 26 & 204 \\
\hline 40 & 81 & 98 & 100 & 85 & 162 & 50 & 529 & 37 & 531 \\
\hline 60 & 121 & 114 & 144 & 109 & 242 & 104 & 841 & 43 & 1037 \\
\hline \multirow[t]{2}{*}{80} & 169 & 138 & 196 & 140 & 338 & 173 & 1521 & 93 & 1588 \\
\hline & & \multicolumn{4}{|c|}{$\alpha=0.8$} & \multicolumn{4}{|c|}{$\alpha=0.8$} \\
\hline$k$ & $N_{\text {sub }}$ & grid CS & $n_{\mathrm{CS}}$ & DtN CS & $n_{\mathrm{CS}}$ & grid CS & $n_{\mathrm{CS}}$ & DtN CS & $n_{\mathrm{CS}}$ \\
\hline 10 & 36 & 19 & 49 & 26 & 72 & 15 & 121 & 10 & 122 \\
\hline 20 & 100 & 35 & 121 & 61 & 200 & 20 & 361 & 13 & 394 \\
\hline 40 & 361 & 88 & 400 & 139 & 722 & 35 & 1369 & 22 & 1440 \\
\hline 60 & 676 & 187 & 729 & 191 & 1352 & 52 & 2601 & 39 & 2700 \\
\hline & 1089 & 331 & 1156 & 250 & 2178 & 78 & 4225 & 68 & 4352 \\
\hline & & \multicolumn{4}{|c|}{$\alpha=1$} & \multicolumn{4}{|c|}{$\alpha=1$} \\
\hline$k$ & $N_{\text {sub }}$ & grid CS & $n_{\mathrm{CS}}$ & DtN CS & $n_{\mathrm{CS}}$ & grid CS & $n_{\mathrm{CS}}$ & DtN CS & $n_{\mathrm{CS}}$ \\
\hline 10 & 100 & 26 & 121 & 52 & 200 & 17 & 324 & 11 & 324 \\
\hline 20 & 400 & 26 & 441 & 43 & 800 & 23 & 1089 & 14 & 1120 \\
\hline 40 & 1600 & 33 & 1681 & 157 & 3200 & 22 & 4624 & 20 & 4640 \\
\hline 60 & 3600 & 45 & 3721 & 338 & 7200 & 26 & 10404 & 29 & 10560 \\
\hline 80 & 6400 & 62 & 6561 & $>500$ & 12800 & 30 & 18769 & 44 & 18880 \\
\hline
\end{tabular}

Table 2: $(d=2)$ Number of iterations (and coarse space size $\left.n_{\mathrm{CS}}\right)$ for the two-level preconditioners with the grid coarse space/DtN coarse space forcing similar $n_{C S}$, with $H_{\text {sub }} \sim k^{-\alpha}, \varepsilon_{\text {prec }}=k$.

automatic choice criterion; note that in $3 d$ the number of selected eigenvectors is larger than in $2 d$, but we only keep a maximum of 20 eigenvectors in each subdomain. In Table 3 we report the results of this experiment. As expected, for the grid coarse space the best iteration counts are obtained for $\alpha=0.5$ because then $\alpha^{\prime}=1$ gives the coarse mesh with the smallest diameter among the experimented ones: the number of iterations grows slowly, with $\mathcal{O}\left(k^{0.61}\right) \cong$ $\mathcal{O}\left(n^{0.13}\right)$. With higher $\alpha$ the iteration counts get worse quickly, and $\alpha=0.8$ is not usable. For the DtN coarse space, the larger coarse space size is obtained by taking $\alpha$ bigger (recall that $\alpha^{\prime}$ is not a parameter in the DtN case): for $\alpha=0.8$ the number of iterations grows slowly, with $\mathcal{O}\left(k^{0.2}\right) \cong \mathcal{O}\left(n^{0.04}\right)$, but this value may be optimistic, there is a decrease in iteration number between $k=20$ and 30 . We believe that for the other values of $\alpha$, where the iteration counts are not much better or worse than with the one-level preconditioner, we did not compute enough eigenvectors in each subdomain to build the DtN coarse space. 


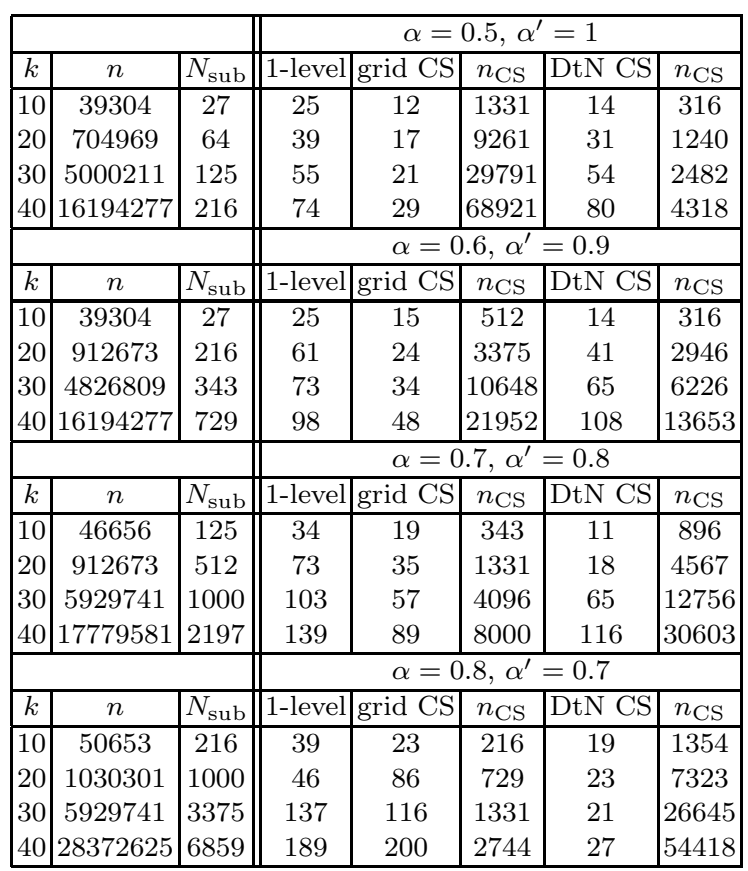

Table 3: $(d=3)$ Number of iterations (and coarse space size $\left.n_{\mathrm{CS}}\right)$ for the onelevel preconditioner and the two-level preconditioners with the grid coarse space/DtN coarse space, with $H_{\text {sub }} \sim k^{-\alpha}, H_{\text {coarse }} \sim k^{-\alpha^{\prime}}, \varepsilon_{\text {prec }}=k$.

\section{Conclusion}

We tested numerically two different coarse space definitions for two-level domain decomposition preconditioners for the pure Helmholtz equation (discretized with piecewise linear finite elements), both in $2 d$ and $3 d$, reaching more than 15 million degrees of freedom in the resulting linear systems. The preconditioners built with absorption $\varepsilon_{\text {prec }}=k^{2}$ appear to perform much worse than those with absorption $\varepsilon_{\text {prec }}=k$. We have seen that in most cases for smaller coarse space sizes the grid coarse space gives fewer iterations than the DtN coarse space, while for larger coarse space sizes the grid coarse space gives generally more iterations than the DtN coarse space. The best iteration counts for the grid coarse space are obtained by separating the coarse mesh diameter $H_{\text {coarse }} \sim k^{-\alpha^{\prime}}$ from the subdomain diameter $H_{\text {sub }} \sim k^{-\alpha}$, taking $\alpha^{\prime}>\alpha$. Both for the grid coarse space and the DtN coarse space, for appropriate choices of the method parameters we have obtained iteration counts which grow quite slowly with the wavenumber $k$. Further experiments to compare the two coarse spaces should be carried out in the heterogenous case. 
Acknowledgement This work has been supported in part by the French National Research Agency (ANR), project MEDIMAX, ANR-13-MONU-0012.

\section{References}

L. Conen, V. Dolean, R. Krause, and F. Nataf. A coarse space for heterogeneous Helmholtz problems based on the Dirichlet-to-Neumann operator. J. Comput. Appl. Math., 271:83-99, 2014.

I. G. Graham, E. A. Spence, and E. Vainikko. Recent Results on Domain Decomposition Preconditioning for the High-Frequency Helmholtz Equation Using Absorption, pages 3-26. Geosystems Mathematics. Springer, 2017a.

I. G. Graham, E. A. Spence, and E. Vainikko. Domain decomposition preconditioning for high-frequency Helmholtz problems with absorption. Math. Comp., 86(307):2089-2127, 2017b.

J.-H. Kimn and M. Sarkis. Restricted overlapping balancing domain decomposition methods and restricted coarse problems for the Helmholtz problem. Comput. Methods Appl. Mech. Engrg., 196(8):1507-1514, 2007. 